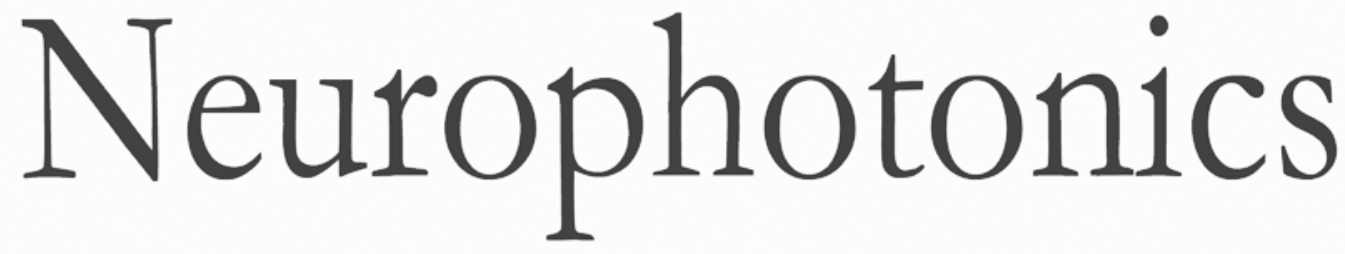

\title{
Measurement, modeling, and prediction of temperature rise due to optogenetic brain stimulation
}

Gonzalo Arias-Gil

Frank Walter Ohl

Kentaroh Takagaki

Michael Thomas Lippert 


\title{
Measurement, modeling, and prediction of temperature rise due to optogenetic brain stimulation
}

\author{
Gonzalo Arias-Gil, ${ }^{a, b}$ Frank Walter Ohl, ${ }^{a, b}$ Kentaroh Takagaki, ${ }^{a, b, \dagger}$ and Michael Thomas Lippert ${ }^{a, *, \dagger}$ \\ aLeibniz Institute for Neurobiology, Systems Physiology of Neuroscience, Brenneckestraße 6, Magdeburg, Saxony-Anhalt 39118, Germany \\ bOtto-von-Guericke University Magdeburg, Faculty for Natural Sciences, Universitätsplatz 2, Magdeburg, Saxony-Anhalt 39106, Germany
}

\begin{abstract}
Optogenetics is one of the most important techniques in neurophysiology, with potential clinical applications. However, the strong light needed may cause harmful temperature rises. So far, there are no methods to reliably estimate brain heating and safe limits in actual optogenetic experiments. We used thermal imaging to directly measure such temperature rises at the surface of live mouse brains during laser illumination with wavelengths and intensities typical for optogenetics. We then modeled the temperature rise with a simple logarithmic model. Our results indicate that previous finite-element models can underestimate temperature increases by an order of magnitude. We validate our empirical model by predicting the temperature rise caused by pulsed stimulation paradigms. These predictions fit closely to the empirical data and constitute a better estimate of real temperature increases. Additionally, we provide a web-based app for easy calculation that can be used as a tool for safe design of optogenetic experiments. ๑ The Authors. Published by SPIE under a Creative Commons Attribution 3.0 Unported License. Distribution or reproduction of this work in whole or in part requires full attribution of the original publication, including its DOI. [DOI: 10.1117/1.NPh.3.4.045007]
\end{abstract}

Keywords: optogenetics; thermal imaging; modeling.

Paper 16052RR received Jul. 21, 2016; accepted for publication Nov. 8, 2016; published online Nov. 30, 2016.

\section{Introduction}

Optogenetics enables precise control of genetically defined cell populations, both excitatory and inhibitory. While the biophysics and genetics of the optogenetic sensors themselves are well studied, much less attention has been paid to the influence of the light used, which is typically in the visible spectrum and may cause unintended effects such as heating and photodynamic damage. These side effects, if not understood and controlled for, could curtail the usefulness of the technique as it is applied to wider experimental and clinical contexts. For example, brain tissue will suffer irreversible damage if heated by 6 to $8 \mathrm{~K},{ }^{1,2}$ a temperature that can easily be attained with laser light stimulation. Physiological changes in neural activity may be observed with temperature increases of around 1.5 to $3 \mathrm{~K} .^{1,2}$

Several studies have addressed the issue of heating caused by optogenetic illumination, primarily with bottom-up finiteelement modeling. ${ }^{3,4}$ Although these models have shed light on the biophysical dynamics and mechanisms of brain heating with laser illumination, they are less suited to predict the actual heating and resulting temperatures in the brain. For example, these models are limited by their assumption of microscopic homogeneity within the brain tissue and rely on biophysical parameters with large error margins, often measured in ex vivo preparations devoid of blood, which is an important absorptive element. Given the large number of elements and parameters simulated in finite-element models, any prediction is sensitive to small inaccuracies in these physical parameters, and final predictive results must always be adjusted experimentally. We have therefore developed an alternative top-down approach by descriptively modeling empirical results and validating and

*Address all correspondence to: Michael Thomas Lippert, E-mail: mlippert@ lin-magdeburg.de

TThese authors contributed equally to this work. elaborating on the resulting empirical equation, instead of attempting to provide bottom-up justification.

Measuring actual brain temperature rises in response to light illumination is also challenging. Even the simple act of introducing a temperature sensor into the brain can be problematic because the sensor itself has unique light absorption characteristics and thermal capacity, which perturb the biophysical phenomenon. Moreover, most sensors are heat conductive and relatively large compared to the tissue area of interest, leading to active cooling of the measured area. Furthermore, given the large heat capacities of these sensors, they take relatively long to equilibrate and cannot react on the fast time scale of typical optogenetic stimulation paradigms. Noncontact methods of temperature measurement have been used to try to circumvent this issue. Magnetic resonance thermometry, in particular, can be used to characterize brain heating ${ }^{5}$ in response to optogenetic stimulation, but while the method has good depth penetration, it provides low spatial and temporal resolution. Optical thermography offers superior resolution and speed, although it is limited to exposed surface areas. In this study, we use a high-speed commercial-grade thermal camera to measure the thermal effects of laser illumination on the brain with commonly used optogenetic wavelengths, intensities, and stimulus lengths. The high resolution in space and time facilitates the derivation of our simple empirical model with minimal degrees of freedom to predict brain heating and cooling after stimulation. We demonstrate that this simple mathematical model can be used to estimate the thermal impact of new stimulation paradigms, either by use of our equation and parameters or by calculating and visualizing through our web-based app.

\section{Materials and Methods}

\subsection{Animals}

Twelve adult male C57BL/6 mice (20-27.25 g) were used in the experiments. Animals were housed in a room with a $12 \mathrm{~h}$ light/ dark cycle (lights on at 6:00 AM) and food and water ad libitum. 
All experiments were done in compliance with the guidelines of the European Community (EUVD 86/609/EEC) and were approved by the local ethics commission of the State of Sachsen-Anhalt.

\subsection{Craniotomy Surgery}

We induced anesthesia by applying urethane (Sigma-Aldrich, St. Louis, USA) intraperitoneally at a concentration of $1.25 \mathrm{~g} / \mathrm{kg}$ of animal weight. Experiments commenced after a deep level of anesthesia was attained and the animal lost all motor reflexes. We also injected $0.1 \mathrm{ml}$ of a $0.2 \mathrm{mg} / \mathrm{ml}$ solution of glycopyrronium bromide (Robinul, $0.2 \mathrm{mg} / \mathrm{ml}$ ) to reduce airway secretions and stabilize the preparation.

Mice were fixed in a stereotaxic frame, and a craniotomy was performed over the right parietal cortex. Drilling was done under saline to prevent excessive heat generation. As a final step, we let the surface of the dura mater dry completely ${ }^{6}$ and used dental acrylic to attach a brass holder to the occipital bone. We performed the experiments at $24^{\circ} \mathrm{C}$ and waited for the surface temperature of the craniotomy to stabilize before we started imaging. Due to slow radiative heat loss, the typical baseline temperature of the exposed brain was approximately $30^{\circ} \mathrm{C}$ during the experiment, in accordance with previous studies. ${ }^{7,8}$ At the end of an experiment, the animals were euthanized.

\subsection{Measurement Setup}

For thermography, we used an Optris PI 230 infrared camera (Optris GmbH, Berlin, Germany). The detector of the camera is an uncooled focal plane array with $160 \times 120$ pixels. The sampling rate is fixed by the camera at $128 \mathrm{~Hz}$. We used a lens that provided a field of view (FOV) of 23 by $17 \mathrm{deg}$. In combination with the sample distance of $20 \mathrm{~mm}$, this setup yields a single pixel resolution of $50.9 \mu \mathrm{m}$. Data acquisition was done through the PI Connect software provided by the camera manufacturer. The camera was calibrated by setting a custom emission factor of 0.95 to match measured optical properties of the brain surface and loading the manufacturer-supplied lens and array calibration file. This procedure matched the reading of our camera to that of a needle probe at equilibrium on the brain. Since the recording could not be triggered externally, blocks that contained all trials of an experiment were acquired. The onset of illumination was then determined from a thermal reference made from the exposed filament of a microlightbulb and placed in the FOV.

For laser illumination, we used five custom optical paths with the following laser sources: three diode-pumped solid-state lasers of $473 \mathrm{~nm}$ [blue, module from Changchun New Industries Optoelectronics Technology Co., Ltd. (CNI)], $532 \mathrm{~nm}$ (green, module from DHOM UltraLasers, Inc.), and $589 \mathrm{~nm}$ (yellow, module from CNI), as well as two diode lasers with wavelengths of $633 \mathrm{~nm}$ (red) and $450 \mathrm{~nm}$ (royal blue). Each laser was collimated into the proximal end of the fiber with an aspheric lens. The clean-cut distal end of the fiber was placed directly in the field of view, as described below. Due to propagation within the fiber, the beam profile in air exiting the fiber approximates a super-Gaussian profile [Fig. 1(g)]. This condition is usually typical for optogenetics experiments with multimode fibers, but might not always be present, e.g., due to coupling angle. To control the timing of illumination, lasers were driven in continuous wave mode and shuttered (SR474; Stanford Research Systems). Light intensity was adjusted by a variable neutral density filter. Typically, we used an illumination intensity of $10 \mathrm{~mW}$ for $500 \mathrm{~ms}$ if not specified otherwise. For experiments that are not wavelength-specific, we used the 450-nm laser because of its high stability.

The camera, timing reference, and delivery fiber tip were all mounted onto a stable optical breadboard together with the anesthetized animal [Figs. 1(a) and 1(b)]. The camera was fixed at a $45 \mathrm{deg}$ angle in relation to the platform and the brain surface. The fiber tip was positioned with a micromanipulator and held at a 45 deg angle in relation to the brain surface. This results in a slightly oval shape of the resulting illuminated spot, but balances spot size distortion and measurement access. Also, a $200 \mu \mathrm{m}$ multimode fiber with a numerical aperture of 0.22 was used. The numerical aperture was fully filled by the lasers.

A measured beam distribution is given in Fig. 1(g), showing the super-Gaussian beam shape as seen from the position of the IR camera. Due to the projection of the fiber aperture onto the brain surface at an angle, power densities in parts of the spot are lower than what would be seen with perpendicular illumination. This might lead to a slight underestimation of actual heating, which we attempted to compensate for by measuring the warmest pixel in the spot. Even though the fiber projection shape is elliptical due to the tilting of the fiber, we can assume an illumination area equal to the fiber tip to calculate power densities of our illumination paradigms, since we are measuring the maximum temperature increase in the illumination area. The approximate area of our $200-\mu \mathrm{m}$ fiber is $0.03 \mathrm{~mm}^{2}$, for the $100 \mu \mathrm{m}$ is $0.008 \mathrm{~mm}^{2}$, and for the $400 \mu \mathrm{m}$ is $0.13 \mathrm{~mm}^{2}$.

\subsection{Measurements and Analysis}

After experiments, data from the PI Connect software were exported as a text file and analyzed in MATLAB 2013a (Mathworks) and Mathematica 10.0.1 (Wolfram Research). The analysis code is available for inspection in Ref. 9. Figures other than Fig. 4 (animal variability) were made with multitrial data sets from individual representative animals to demonstrate qualitative differences.

Trials were segmented from raw data based on thresholding the timing reference temperature. The baseline mean temperature was subtracted to obtain the relative temperature increase. We then conducted nonlinear model fits to various empirical models as described below. Bootstrapping was conducted within trials to estimate the variability of our model fits for individual experiments. To assess model fits, we calculated $R$-squared metrics. To assess statistical differences between wavelengths, we used a Wilcoxon signed-rank test for the parameter values, where a $p$-value $<0.05$ was considered a significant result. All analyses, figures, and tables for the results section were generated using standard Mathematica functions.

\section{Results}

\subsection{Deriving a Parametric Model to Fit Thermographic Recordings}

Upon laser illumination, a clear temperature rise can be seen in the several camera pixels sampling the heated area [Fig. 1(c)]. This area of elevated temperature decays rapidly with spatial distance from the heated area. Since the heated area only covers several pixels and we are primarily interested in the maximum temperature rise due to illumination, we assumed a local pointlike process at the tip of the illumination fiber and took the 
(a)

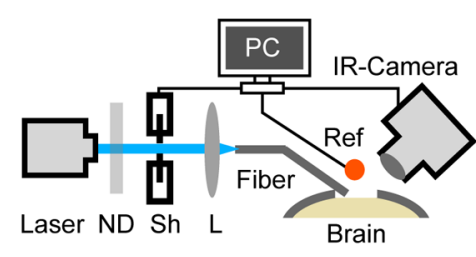

(b)

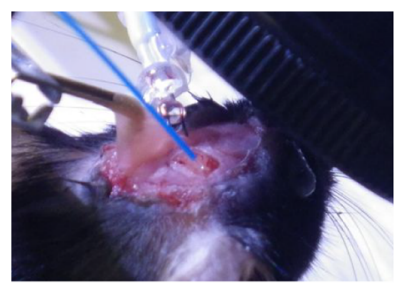

(c)

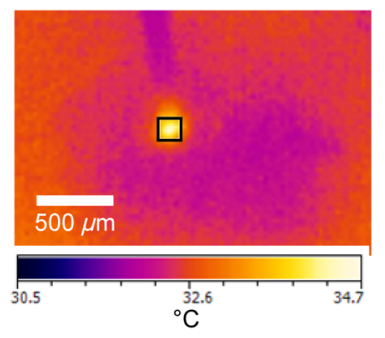

(d)

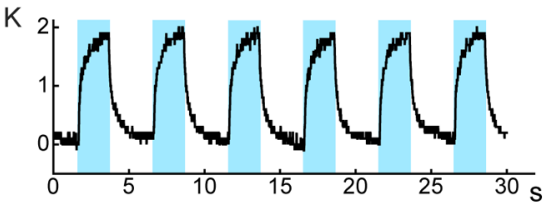

(e)

$$
\begin{aligned}
& \mathbf{f}_{\text {rising }}(t)=\mathbf{a} \log (\mathbf{b}+\mathbf{c} t) \\
& \mathbf{f}_{\text {taling }}(t)=\mathbf{g}_{1} \mathbf{e}^{\mathbf{h}_{1} t}+\mathbf{g}_{2} \mathbf{e}^{\mathbf{h}_{2} t}
\end{aligned}
$$

(g)

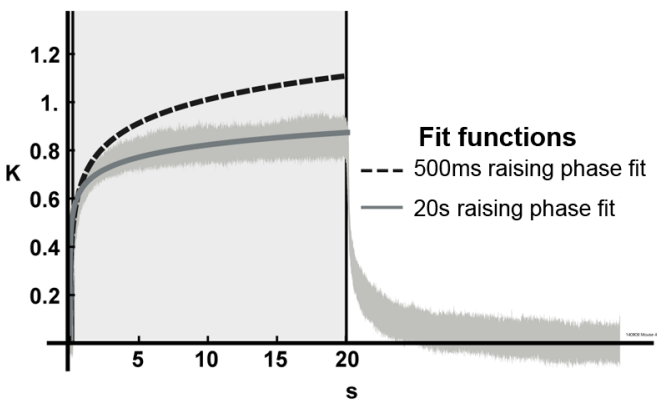

(f)

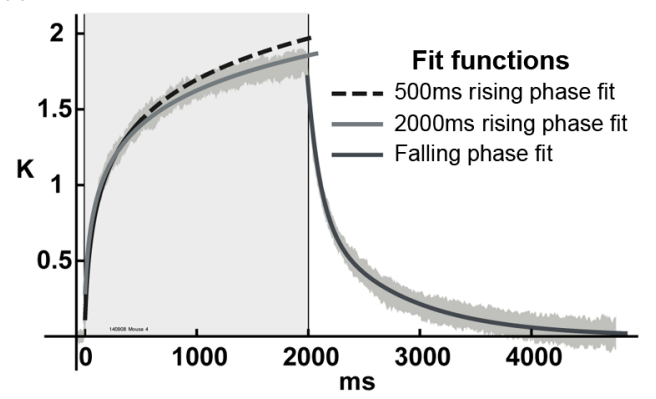

(h)

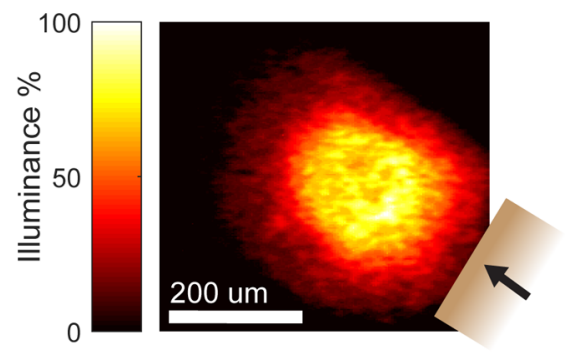

Fig. 1 Overview of the setup, measurements, and our mathematical model describing temperature variation. (a) Schematic representation of our setup. Light is emitted by a laser, attenuated by a variable neutral density filter (ND), gated by a blade shutter (Sh), and focused into the fiber by a collimation lens (L). The brain is imaged by an IR-camera system. A small thermal reference is placed within the field of view to trigger acquisition events (Ref). (b) Close-up view of the craniotomy; the fiber is placed on top of the exposed surface of the brain and the illuminated spot is imaged with the IR-camera. (c) Thermography image of the craniotomy during laser illumination. The craniotomy is distinguishable from the surrounding bone by its difference in thermal emission. (d) Example of a temperature-time diagram with illumination pulses of $2000 \mathrm{~ms}$; blue rectangles indicate timing of laser illumination. (e) Equations corresponding to the rising phase fit function and the falling phase fit function. The rising phase was fitted with a logarithmic model that is defined by three parameters: a (scaling), $\mathbf{b}$ (timing adjustment), and $\mathbf{c}$ (shape). The falling phase was fitted with an exponential decay model, where $\mathbf{g}_{1}$ and $\mathbf{g}_{2}$ are the magnitude parameters and $\mathbf{h}_{\mathbf{1}}$ and $\mathbf{h}_{\mathbf{2}}$ are the time parameters. (f) Measured data (mean \pm standard deviation in gray) and model fits. The dashed line corresponds to the extrapolated prediction of a model obtained by fitting only the first $500 \mathrm{~ms}$ worth of data for the rising phase ( $n=35$ trials). (g) Measured data (mean \pm standard deviation in gray) and model fits for an illumination time of $20 \mathrm{~s}$. The dashed line corresponds to the extrapolated prediction of a model obtained by fitting only the first $500 \mathrm{~ms}$ worth of data for the rising phase $(n=38$ trials). (h) Beam shape and relative irradiance from $200-\mu \mathrm{m}$ fiber recorded through the IR camera.

maximal pixel temperature in the region of interest as a measure of the heated tissue temperature. We avoided taking the mean temperature of the region of interest, since border pixels only partially covering the illuminated/heated area would dilute the mean and lead to a false underestimate. The temperature rise in response to heating appears at first glance to be exponential, as some models seem to predict. ${ }^{4}$ Upon closer inspection, however, we found that regardless of the measurement duration within our time frame of up to $2000 \mathrm{~ms}$, the temperature would continue to rise and not equilibrate [Fig. 1(f)]. We selected the simplest mathematical model matching these characteristics: a logarithmic rise, scaled by an amplitude constant $\mathbf{a}$ and a time constant $\mathbf{c}$ :

$\mathbf{f}_{\text {rising }}(t)=\mathbf{a} \log (\mathbf{b}+\mathbf{c} t)$.

The constant $\mathbf{b}$ was included to account for trigger timing jitter (given the relatively low temporal sampling rate of thermal cameras compared to the rapid speed of initial temperature rise) and to improve fitting accuracy. Under ideal measurement conditions, this constant would, by definition, be 1 . 
To evaluate this model qualitatively, we first recorded from individual animals without pooling the data (to avoid interanimal variability, which is discussed later). A total of 40 trials of illumination were recorded per animal with a 450-nm laser at $5 \mathrm{~mW}$ optical power for $2 \mathrm{~s}$. The standard sample number for each illumination paradigm throughout this report will be 40 trials per animal unless specified otherwise. As can be seen in Fig. 1(e), our logarithmic model fits the temperature rise over $2000 \mathrm{~ms}$ exceptionally well ( $r^{2}$-goodness of fit: 0.997$)$. Fitting the same temperature rise during the initial $500 \mathrm{~ms}$ interval still results in a good fit when extrapolated out to $2000 \mathrm{~ms}$ worth of data $\left(r^{2}=0.943\right)$, with improved precision for the earliest part of the temperature increase. Of note, this earliest phase of temperature rise features kinetics that are fast compared to the $7 \mathrm{~ms}$ sampling interval of our camera and, thus, are subject to more error and instability in model fitting than the later, more stable phases of rise. During subsequent experiments, the fit interval was tailored to the desired pulse and train lengths. Increasing the illumination to the time scale of tens of seconds produces the same results as seen in Fig. 1(g). Our model is able to fit an illumination time of $20 \mathrm{~s}$, meaning the temperature trend is still logarithmic; this should not change for any illumination time, as long as the temperature increase is within the range where brain heating does not cause irreversible changes in tissue biophysical characteristics. The fit of the initial $500 \mathrm{~ms}$ also results in a reasonable prediction when extrapolated to the full $20 \mathrm{~s}$, and the final predicted temperature increase lies well within our variability interval.

The temperature fall during the cooling phase shows an exponential-like time course. In contrast to the rising phase, which is logarithmic, the falling phase is indeed exponential with a limit equilibrium tissue temperature of the surrounding tissue (in this case, the exposed craniotomy field). A simple exponential did not fit the data sufficiently well, especially in the initial rapid fall, but a double-exponential provided excellent fits (Fig. 1, $r^{2}$-goodness of fit: 0.997). Based on this finding, we propose the following empirical model:

$\mathbf{f}_{\text {falling }}(t)=\mathbf{g}_{1} e^{\mathbf{h} 1 t}+\mathbf{g}_{2} e^{\mathbf{h} 2 t}$,

where $\mathbf{g}_{\mathbf{1}}$ and $\mathbf{g}_{\mathbf{2}}$ are the scaling constants and $\mathbf{h}_{\mathbf{1}}$ and $\mathbf{h}_{\mathbf{2}}$ are the two time constants. The approximate order of magnitude of these two decay constants was generally -0.01 and -0.001 , respectively, resulting in exponential time constants on the order of 100 and $800 \mathrm{~ms}$. Since the focus of our study was on maximum safety limits and the precise decay function constants seemed to show complex dependence upon heating history, we defer further discussion of this falling phase to future reports.

\subsection{Influence of Illumination Intensity on Brain Heating}

Increased illumination strength naturally causes increased heating of the tissue. We first tested this qualitative relationship by exposing the brain of a single animal to several intensities within the range used in optogenetic experiments $(2.5,5,7.5$, and $10 \mathrm{~mW}$ ) and by measuring and modeling the resultant temperature increases [Figs. 2(a) and 2(b)]. By changing the illumination power coming out of a fiber with the same diameter $(200 \mu \mathrm{m})$, we are effectively changing the power density of the illumination and therefore studying its effects on brain heating. For the given intensities, the respective power densities amount to $83.3,166.6,250$, and $333.3 \mathrm{~mW} / \mathrm{mm}^{2}$. The fiber tip was placed in a blood vessel free area at the beginning of the experiment, and its position was kept constant to avoid introducing variability from differences in blood vessel density on the surface of the brain. The same procedure was followed in all experiments, except for those in which deviations were inevitable, such as when we measured the influence of fiber diameter.

A linear increase in temperature with power indicates that energy influx dominates the final temperature outcome, whereas a nonlinear relationship would indicate a substantial dynamic involvement of other processes such as cooling. A priori, one would expect the former situation given the massive amount of power input from illumination (e.g., $10 \mathrm{~mW} / 0.03 \mathrm{~mm}^{2}$ would amount to a power input of $33 \mathrm{~W}$ in $1 \mathrm{~cm}^{2}$, comparable to the total power of an incandescent desk lamp or small soldering iron). As expected, our results show a linear increase in temperature with illumination intensity or power density [Fig. 2(a)]; this linear increase is reflected in our model parameter a but not on model parameters b or c [Fig. 2(b)].

During exposure to $10 \mathrm{~mW}$ of $450 \mathrm{~nm}$ light for $500 \mathrm{~ms}$, the brain heats up to an average of $6.35 \mathrm{~K}$ above baseline $(200-\mu \mathrm{m}$ fiber). This would encroach upon the maximal tolerable temperature increase without permanent tissue damage (6 to $8 \mathrm{~K})$. $^{1,2}$ Out of the calculated model parameters, the a parameter increases linearly with power, while the shape parameter c (as well as the timing adjustment parameter b) remain largely unaffected [Fig. 2(b)].

\subsection{Influence of Illumination Wavelength on Brain Heating}

The interaction of light with tissue and transfer of heat energy is mainly determined by two separate physical processes: scattering and absorption. Scattering is mainly due to heterogeneities in the refractory index within nervous tissue. Absorption in a relatively transparent tissue such as the brain is governed, to a significant extent, by hemoglobin. Both processes cause a wavelength dependence of heat transfer to the tissue. Scattering increases exponentially at shorter wavelengths, ${ }^{10,11}$ but absorption is complex and depends mainly on the hemoglobin absorption spectrum. In the visible light range, which is used in optogenetics, it generally decreases with wavelength.

To establish a quantitative relationship between various wavelengths and heating, we tested four additional wavelengths typically used in optogenetics [Figs. 2(c)-2(f)]: blue (473 nm), green $(532 \mathrm{~nm})$, yellow $(589 \mathrm{~nm})$, and red $(633 \mathrm{~nm})$. In this experiment, we used data obtained from eight animals to obtain global estimates of parameter values for the predictive equation and for our web-based calculation app to predict brain heating due to optogenetic illumination. For every animal, we measured the temperature increase during 40 trials for each of the four previously mentioned wavelengths, illuminating at $10 \mathrm{~mW}$ of power for $500 \mathrm{~ms}$ through a fiber of $200-\mu \mathrm{m}$ diameter. The largest temperature increase in a typical animal was seen with blue and green light [Figs. 2(c) and 2(d); blue 1.39 K, green 1.34 K). Yellow light caused a medium increase $(0.91 \mathrm{~K})$ and red light caused the smallest increase $(0.33 \mathrm{~K})$. This is directly reflected in the values of the first $(Q 1)$ and third $(Q 3)$ quartiles for the a parameters of the fits [blue 0.39 to 0.771 , green 0.418 to 0.808 , yellow 0.287 to 0.664 , and red 0.077 to 0.271 ; Fig. $2(\mathrm{~g})$ ]. The c parameters showed a similar trend for these wavelengths [blue: 0.02 to 0.097 , green: 0.026 to 0.118 , yellow: 0.02 to 0.088 , red: 0.017 to 0.054 ; Fig. $2(\mathrm{~g})$ ], which is likely a manifestation of deeper penetration of longer wavelengths. 
(a)

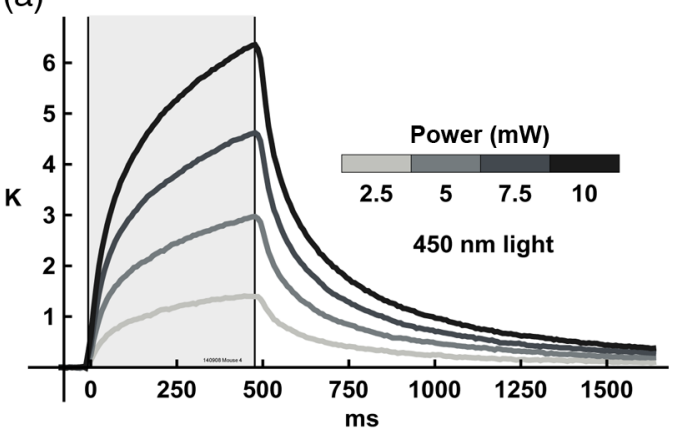

(c)

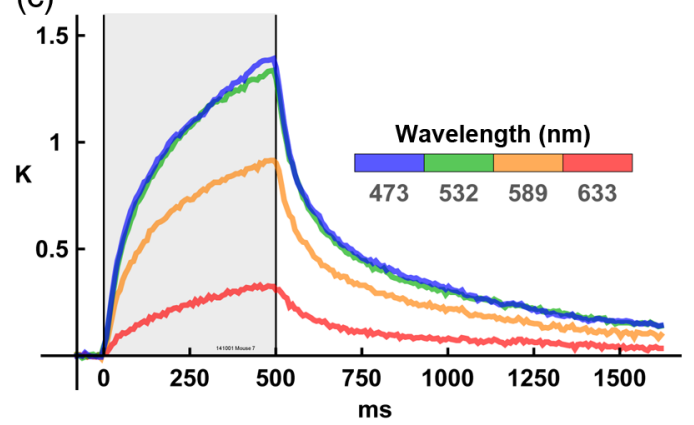

(e)

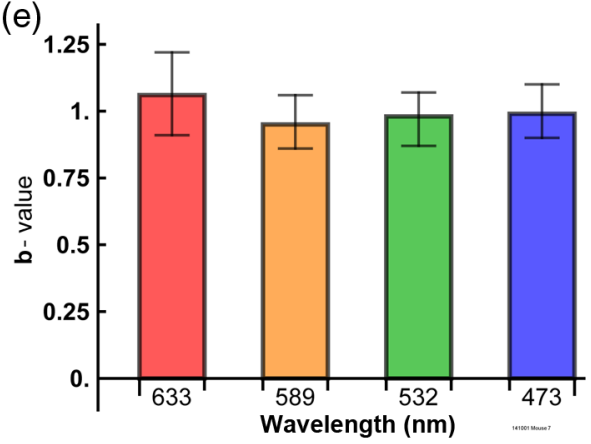

(b)

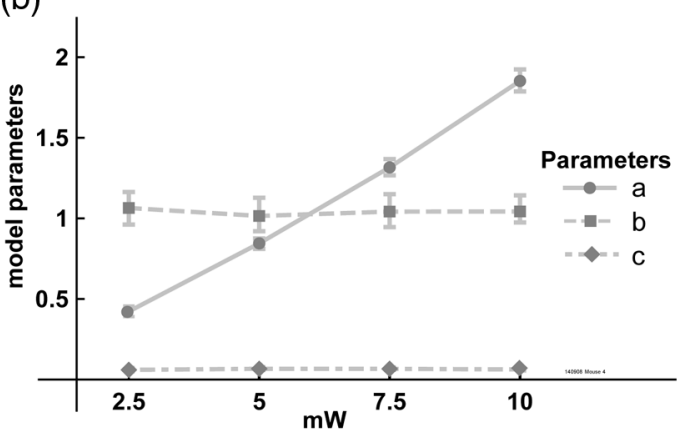

(d)

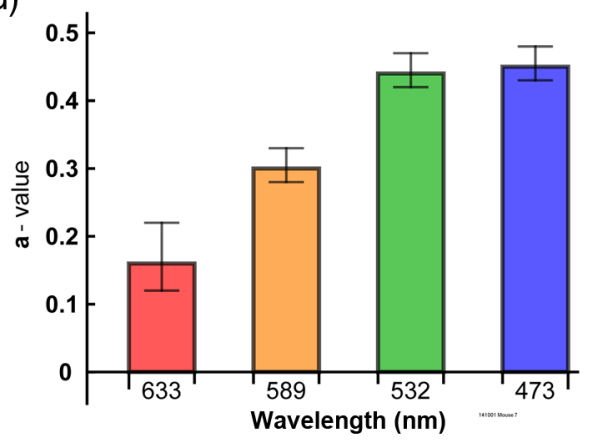

(f)

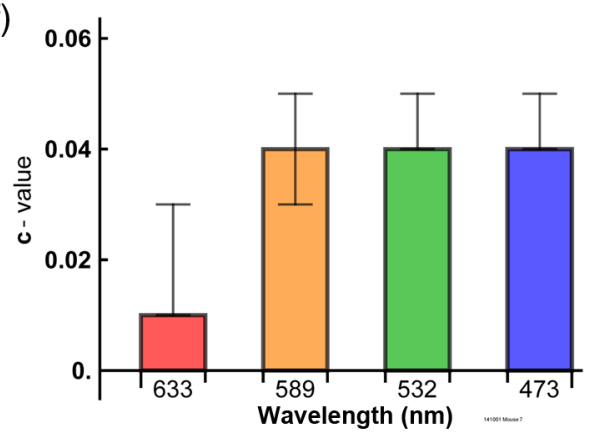

Fig. 2 Effects of the illumination power and wavelength. (a) Increased illumination power leads to higher temperature changes with similar time profile at $450 \mathrm{~nm}$ (means from $n=38$ trials per power value). (b) A linear increase of the a parameter of our model reflects these changes; the shape parameter $\mathbf{c}$ remains constant. Error bars correspond to the $1 \%$ to $99 \%$ confidence intervals from approximately $1: 4$ bootstrapped data. (c) Temperature increase profile for different wavelengths of laser light $(10 \mathrm{~mW})$. Different temperature change trends are seen (means from $n=40$ trials per wavelength). (d-f) Different wavelengths differentially affect the model $\mathbf{a}$ and $\mathbf{c}$ parameters, representing different absorption magnitude and kinetics. The remaining $\mathbf{b}$ parameter remains around a fixed value of 1 , as explained in the text. Data for eight animals ( 40 trials per animal and per wavelength) are represented as a box-andwhiskers plot showing the median, quartiles, and maximum/minimum values for each wavelength. The black dots correspond to mean values ( 40 trials) for the parameters for each wavelength and animal. The parameter values for different wavelengths corresponding to the same animal are connected with gray lines. Significant differences between parameter values of different wavelengths are marked with a star ( $p$-value $<0.05$, Wilcoxon signed-rank test). Table showing the median as well as the first $(Q 1)$ and the third quartiles (Q3) for $\mathbf{a}, \mathbf{b}$, and $\mathbf{c}$ parameters at all studied wavelengths.

To assess the statistical significance of this qualitative result, we performed a Wilcoxon signed-rank test for the parameter values in which a $p$-value $<0.05$ was considered significant. This test was chosen given the asymmetric and nonnormal nature of our data set, which is mainly due to two outlier samples. For parameter a, we obtained significant differences between red and yellow, red and blue, red and green, yellow and green, and, finally, yellow and blue [Fig. 2(d)]. Although the trend was similar to the values for the c parameter, only the differences in yellow and green wavelengths were significant based on our statistical assumptions [Fig. 2(d)].

\subsection{Influence of Fiber Diameter on Brain Heating}

Next, we tested the effect of fiber diameter on heating. Thinner fibers achieve higher local power density and are therefore expected to induce more local heating, as indicated by our results regarding the effects of illumination intensity on the brain heating. In the most ideal circumstances, we would expect the brain heating to be scaled by the power density, which is inversely proportional to the illumination area and inversely proportional to the square of the fiber diameter. However, this linearity might not hold at different fiber diameters due to factors 
such as the surface/volume-relationship of the illuminated spot or the increased probability of illuminating a significant blood vessel with larger fiber diameters.

To test this hypothesis experimentally, we used three different fiber diameters $(100,200,400 \mu \mathrm{m})$ with constant input numerical aperture and constant power $(5 \mathrm{~mW})$, with a fixed wavelength $(450 \mathrm{~nm})$ (Fig. 3). For each fiber diameter, the respective power densities are $625,166.6$, and $38.5 \mathrm{~mW} / \mathrm{mm}^{2}$. We performed the measurements in one animal and recorded 40 trials for each of the fiber diameters. Due to slight changes in the position of the fiber tip during fiber exchange, some additional variability is to be expected in these measurements. We found the largest temperature increase for the $100-\mu \mathrm{m}$ fiber $(1.79 \mathrm{~K})$ but no difference between 200 and $400 \mu \mathrm{m}(1.48$ and $1.49 \mathrm{~K}$, respectively). This violates the predicted effect due to differences in power density; this is likely due to the unavoidable illumination of blood vessels with larger fiber sizes. The a parameter of the model did not differ between fiber diameters, but the $\mathbf{c}$ parameter decreased with the diameter [Figs. 3(b) and 3(c)], indicating a change in the heating process, e.g., less local heating.

For the larger diameters, especially $400 \mu \mathrm{m}$, it was difficult to avoid larger surface vessels. To investigate this confound, we compared temperature rise with a $200-\mu \mathrm{m}$ fiber placed both (a) directly above and (b) as far as possible from large surface blood vessels. We found a large difference in temperature rise: direct illumination of the vessel for $500 \mathrm{~ms}$ with a 450-nm wavelength laser at $5 \mathrm{~mW}$ of power caused a twofold increase in temperature $(1.22 \mathrm{~K})$ over illumination away from visible blood vessels (0.66 K) [Fig. 4(a)]. We take this twofold difference as an index of the variability of heating due to the presence or absence of blood vessels. This increase in heating with blood vessel illumination suggests that the heat diffusing capacity of blood is negligible compared to its increased energy absorption. The effect is linked to a change in the $\mathbf{c}$ parameter of our model [Fig. 4(b)].
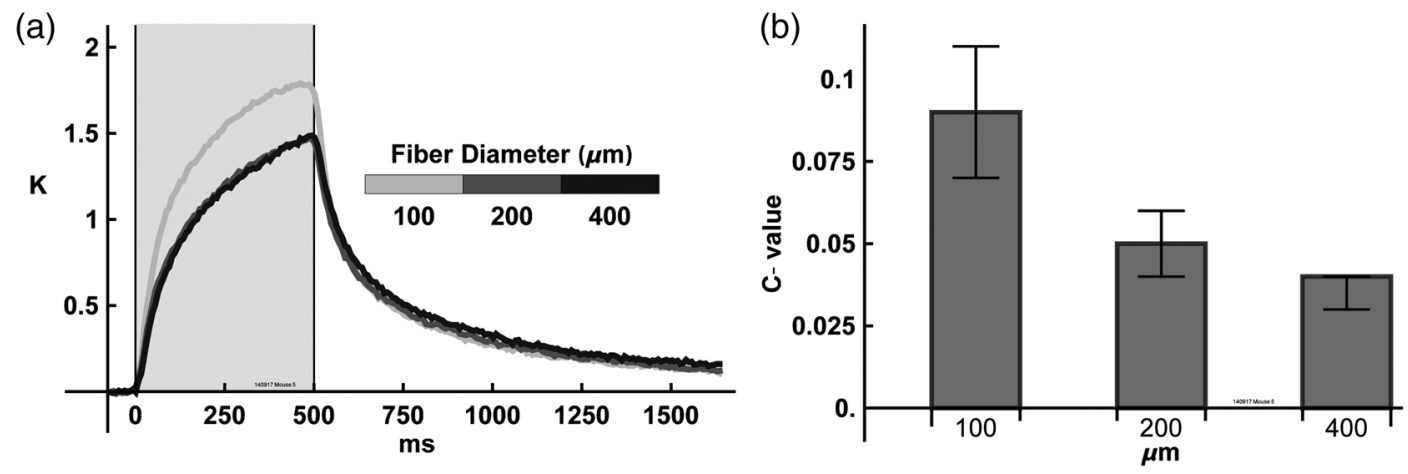

(c)

\begin{tabular}{|c|c|c|c|}
\hline FiberDiamer $(\mu \mathrm{m})$ & 100 & 200 & 400 \\
\hline a $(1 \% \mathrm{Cl}-99 \% \mathrm{Cl})$ & $0.49(0.47-0.52)$ & $0.47(0.43-0.49)$ & $0.50(0.48-0.52)$ \\
\hline b $(1 \% \mathrm{Cl}-99 \% \mathrm{Cl})$ & $1.02(0.88-1.23)$ & $1.06(0.95-1.21)$ & $1.01(0.93-1.10)$ \\
\hline c $(1 \% \mathrm{Cl}-99 \% \mathrm{Cl})$ & $0.09(0.07-0.11)$ & $0.05(0.04-0.06)$ & $0.04(0.03-0.04)$ \\
\hline
\end{tabular}

Fig. 3 Effect of fiber diameter on heating. (a, b) Thinner fibers have higher local irradiance and therefore induce more local heating, in general. No clear difference is seen between 200 and $400 \mu \mathrm{m}$ fibers, likely due to the unavoidable illumination of blood vessels with larger fiber sizes (see text). Plots and error bars are as in Fig. 2. (c) Table representing the model fit values. Changes are mainly seen in the c parameter. 
(a)

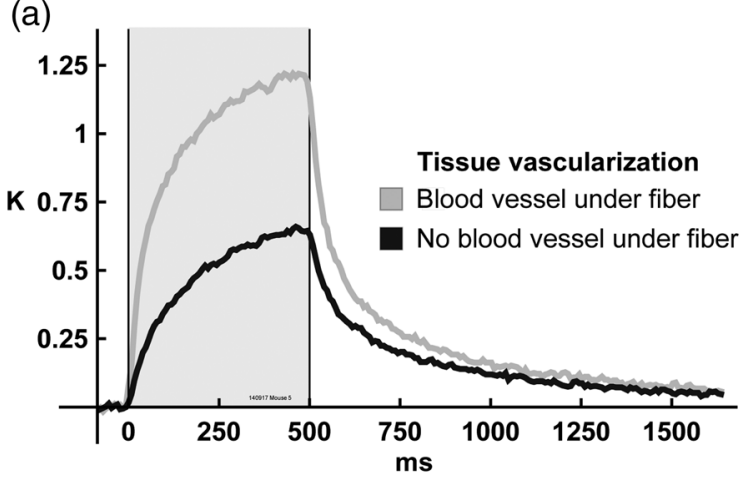

(c)

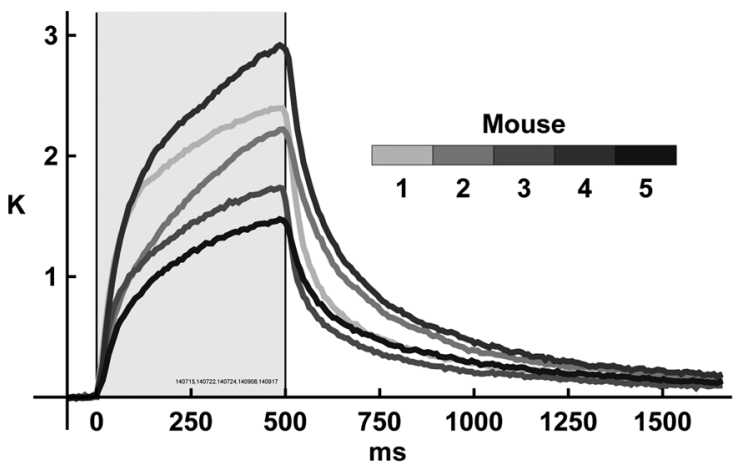

(b)

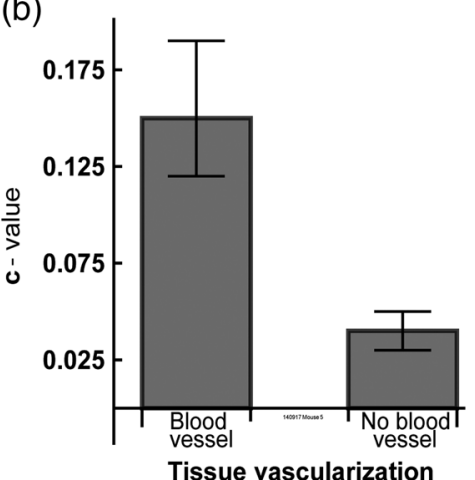

(d)

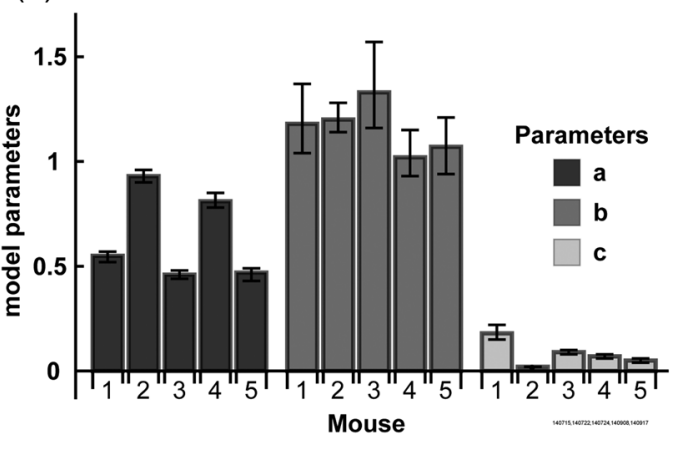

Fig. 4 Biological variability in brain heating. (a) Differences in the vascularization of the illuminated tissue have a significant effect on the temperature increase. Illumination of an area containing a visible blood vessel leads to twofold higher temperatures than illumination of an adjacent area without a vessel (means from $n=37$ to 40 trials each). (b) Vascularization leads to a change in the c parameter of our model, indicating different absorption kinetics. Error bars are as in Fig. 2(b). (c) Interanimal variability from five different mice imaged under the same conditions, as discussed in the text (means from $n=37$ to 40 trials per animal). (d) This interanimal variability in heating is reflected in large differences in the a and c parameters of the model indicating different magnitude and kinetics of absorption, similar to the variability seen in (a). The $\mathbf{b}$ parameter remains around a fixed value of 1 , as explained in the text. Error bars are as in Fig. 2(b).

train. For example, in a $10 \mathrm{~Hz}$ stimulation paradigm with $10 \mathrm{~ms}$ pulses that gives a $10 \%$ duty cycle, we scaled the value for the model parameter a to $10 \%$ of that obtained with constant illumination. We tested both yellow $(589 \mathrm{~nm})$ and blue $(473 \mathrm{~nm})$ light, and found a good match between prediction based on duty cycle and actual measured data from pulsed illumination [Figs. 5(a) and 5(b)]. Although this method does not allow the precise prediction of instantaneous maximum temperatures, the method is robust and does provide an estimate of pulsed temperatures across the frequencies tested.

In principle, we can use our rising and falling phase equations [Fig. 1(e)] in combination to predict the instantaneous pulsed temperature time course (i.e., individual time points along the sawtooth-like temperature trajectory). However, in practice, we find that this leads to systematic accumulating overestimation of the temperature, which we attribute to heating history effects that are not fully accounted for by our current falling phase model. For the purposes of the current report, the duty cycle scaling gives accurate results overall, and since the pulse paradigms commonly used do not feature higher duty cycles, the sawtooth-like peaks are not significantly higher than the overall temperature rise trend (Fig. 5). However, the scaled values should be interpreted in light of this slight underestimate of instantaneous maximum temperature. We intend to investigate this issue further in detail in a report to follow.

\subsection{Web and Smart Phone Interface for Easy Predictions}

As described previously, one main goal was to provide an empirical model with which one can predict brain heating to assist in the design of optogenetic experiments (Fig. 1). To simplify this process, we provide a web-based calculation app, which can also be used on smart phones. ${ }^{12}$ This can be used to predict an approximate safety limit for cortical illumination, but for other tissues, histological differences in absorbance and other characteristics may change model parameters and limit actual predictability. For further caveats, see Sec. 4.

\section{Discussion}

We describe a method to empirically estimate the thermal influence of optogenetic brain stimulation based on results from surface infrared thermography and modeling.

The use of infrared thermography offers several advantages: (1) the heated tissue itself acts as a sensor and is therefore not perturbed by the measurement, (2) temporal resolution is higher than other methods of temperature measurement, and (3) a spatially resolved image is available, which facilitates precise targeting of the measurement site. Furthermore, due to industrial uses, thermal cameras have become affordable. These advantages make it easy to image and test the temperature rise resulting from 

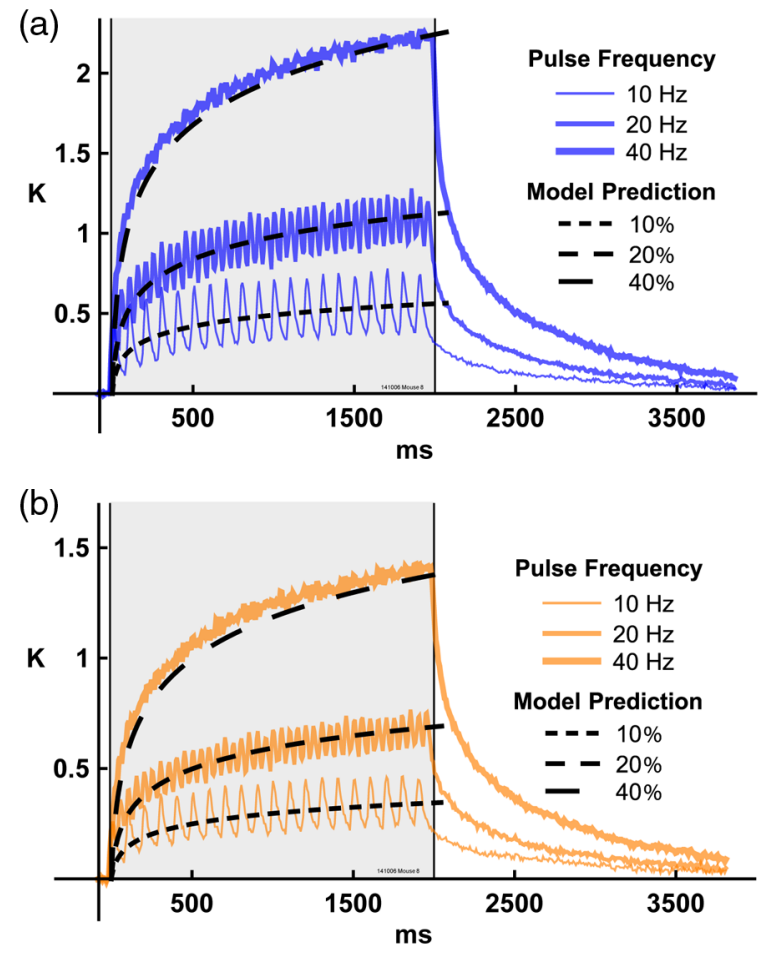

Fig. 5 Heating from pulsed illumination can be approximated by duty cycle scaling. (a) The average temperature profiles for different frequencies of pulsed illumination are plotted in blue $(473 \mathrm{~nm}$, $20 \mathrm{~mW}, 10 \mathrm{~ms}$ pulses; means from $n=33$ to 38 trials per pulse frequency). The model was fitted as previously to data from constant illumination (fit from $n=39$ trials) and scaled by the respective duty cycles of the pulse trains (black dashed lines). (b) Data and fit prediction similar to (a) for yellow light illumination $(589 \mathrm{~nm}$; means from $n=38$ to 39 trials per pulse frequency; model fit from $n=$ 39 trials). For both wavelengths, the actual temperature increase from pulsed illumination is well approximated by scaling the fitted constant illumination model. For example, delivering $10 \mathrm{~ms}$ pulses at $10 \mathrm{~Hz}$ results in a $10 \%$ duty cycle, and the heating is accurately predicted by scaling the constant illumination model by $10 \%$.

a stimulation paradigm before using it in a larger group of animals or patients.

As with any method, thermography has properties that must be taken into account when interpreting results. The most pronounced feature is confinement to measuring surface structures. This limits the range of tissue types that can be imaged, an important factor when considering the large variety of optical properties present in different brain structures. ${ }^{11,13}$ However, it is possible to expose the structures of interest to partially circumvent this problem or to measure exposed structures with similar anatomical/optical properties (e.g., measuring on corpus callosum to estimate white matter temperature rise). In this regard, we take advantage of the relatively homogeneous and exposed nature of the rodent cerebral cortex.

Surface measurements also result in the tissue being exposed to meninges/air on one side. This will lead to radiative heat loss to the environment, potentially leading to an underestimation of temperature rise. In an intracranial location, by contrast, the heated tissue is encircled by tissue on all sides, leading to better heat dissipation via conduction and perfusion but not by radiation (but see Kalmbach and Waters ${ }^{7}$ for different heat dynamics on a longer time scale). Assuming that the missing hemisphere of heat-dissipating brain tissue has similar characteristics to the tissue investigated in our experiments and that air is much less conductive of heat, our estimates of temperature rise would be an overestimate of at most twofold, slightly reduced by the radiative losses, which are, however, minimal on the subsecond time scale. A further source of slight underestimation is the increase in spot size of the light by tilting the fiber at $45 \mathrm{deg}$ to gain imaging access. These caveats notwithstanding, we believe that our biologically based estimates do allow estimation of a generally accurate (albeit less precise) safety limit. In general, despite the trend toward underestimation rather than overestimation, our results show much stronger heating as predicted by other studies.

Variability of measurements between animals must also be taken into account when interpreting our results. The variability of our measurements in different animals for a given illumination paradigm is high, with the highest temperature peak recorded being more than double the value of the lowest peak (see Fig. 4). This interanimal variability could be due to various sources: variability in anatomical brain composition, variability in measurement precision (camera and fiber angles), variability in baseline/external temperature, and, as referred to in Sec. 3, variability in vascularity of the irradiated area. We used genetically homogeneous head-fixed mice on heating pads to minimize the first three factors, leaving the final factor, vascularity of the illuminated area, as a likely key contributor. Since the maximum variability between animals [approximately twofold, Fig. 4(c)] has a similar magnitude as the variability between differently vascularized areas [Fig. 4(a)], we therefore attribute the intersubject variability mainly to tissue vascularity. We further suspect that the distinctly higher heating in two outlier animals during the wavelength comparison experiment (Fig. 2) was due to this effect of tissue vascularization, although we lack comprehensive photodocumentation to firmly establish this point.

Given the approximately twofold upper bound overestimate for errors due to heat dissipation and the approximately twofold lower bound underestimate for errors due to vascularity, in combination, estimates based on our results should be of the correct magnitude as a safety factor to ensure safe irradiation in cortical optogenetic illumination paradigms. In addition, the documented variation among animals is an important factor to consider in experimental design of optogenetic studies in general. Such differences are likely present in most optogenetics preparations but are not predicted by conventional modeling based on homogeneous tissue parameters.

Although previous studies have also attempted to measure temperature variation in neural tissue with optogenetic illumination, the inherent variability of prior measurements and their lack of parametric measurements make it very difficult to compare numerical values between studies, thus underscoring the importance of multimodal studies on the subject of laserinduced heating.

Nevertheless, some studies provided sufficient information to allow approximate comparison with our results. For example, in a recent paper, Stujenske et al. ${ }^{4}$ measure a peak temperature increase of $2.2 \mathrm{~K}$ after $30 \mathrm{~s}$ of illumination with a green 532-nm laser at $10 \mathrm{~mW}$ of power through what is likely a $200-\mu \mathrm{m}$ diameter optical fiber. For this purpose, they use a thermistor probe placed antiparallel to the optical fiber. Interpolation into their measured graph of temperature increase [Figs. 3(d) and 3(e)] should give an approximate temperature increase of $0.216 \mathrm{~K}$ at $500 \mathrm{~ms}$. Our actual measurements for a comparable illumination paradigm with a green 532-nm laser for $500 \mathrm{~ms}$ illumination 
duration show approximately $1.4 \mathrm{~K}$ of temperature increase [Fig. 2(a)]. These differences of one order of magnitude for in vivo measurements might be due to the fact that the thermal probe used by Stujenske et al. ${ }^{4}$ could be acting as a heat sink for the illuminated area; hence, the lower values of temperature increase. With blue illumination at $450 \mathrm{~nm}$, their Monte Carlo simulation script provided as a supplement to their study leads to a maximal heating estimate of $0.0581 \mathrm{~K}$. Our comparable measurements with blue $450 \mathrm{~nm}$ standard condition in Fig. 4 (500 ms, $5 \mathrm{~mW}$ illumination with a $450-\mathrm{nm}$ laser through a $200-\mu \mathrm{m}$ fiber with NA 0.22 ), led to approximately $2 \mathrm{~K}$ temperature increase [Fig. 4(c)]. Even considering the previously explained variability, it is surprising that our actual measurements give an almost sevenfold $(532 \mathrm{~nm})$ and 30 -fold $(450 \mathrm{~nm})$ higher increase compared to the Monte Carlo model predictions.

These discrepancies may be due to many factors. First, Stujenske's Monte Carlo simulation does not take into full account the presence of blood vessels in the tissue, which can increase the absorbance dramatically ${ }^{14}$ (Fig. 4). Second, their use of a thermal probe with high heat conductance might have led to lower tissue temperature increases in their measurements, which may not have been sufficiently offset by their extensive static calibrations, especially in the fast subsecond time scale. In addition, a slight offset between fiber tip and sensor might have contributed to lower measured temperatures, since thermistors cannot provide spatially resolved measurement. Finally, their model is based on biophysical parameters extracted from ex vivo tissue. As we demonstrate in Fig. 6, tissue heating changes markedly with the death of the animal and as time passes after death, so these differences may also cause systematic errors in the estimate. All of these factors except the final one would tend toward underestimation of temperature increases.

Compared to this bottom-up modeling approach, which requires a large number of presuppositions, our model takes an empirical approach based on measured data, taking into account the variability between animals as actually measured. As long as one stays within the constraints of experimental assumptions and measurement assumptions, our method has no further a priori assumptions and can therefore give a much more meaningful estimate of brain heating than the Monte Carlo approach, which has poorly defined assumptions that clearly do not hold, as described above. The simple fact that our model can give estimates of animal-to-animal variability that are clearly existent experimentally, but are wholly missing from the Monte Carlo approach, further supports the superiority of our empirical approach.

Our measurements only have a potential overestimation of up to approximately twofold due to the surface measurement, as discussed above. All other circumstances of thermography would lead to a slight underestimation of temperature, e.g., subsampling artifacts, optical pathway losses, increased illumination spot size, oblique laser irradiation, radiative losses, and surface reflectance of cold surround, ${ }^{15}$ which supports the significance of our finding that heating is much more of an issue than previously reported.

There is prior literature that suggests a linear correlation between illumination power and peak temperature increase, ${ }^{4,16}$ similar to what we report here. The linear relationship between power and temperature increase is to be expected under conditions where the heat loss during illumination is small compared to the applied power. In particular, it suggests that the contribution of radiative heat loss, which is proportional to the fourth power of the temperature and an important concern during thermography measurements, is small compared to the heating under our measuring conditions. One study did not find a linear correlation between power and heating (Fig. 3 from McAlinden et al. ${ }^{8}$ ); this is surprising given theoretical considerations and may potentially be due to light-emitting diode (LED) heating and lower LED efficiency at higher light intensities. There is also evidence predicting the heat accumulation in pulsed illumination paradigms, which we describe. ${ }^{17}$

Safe temperature increases from the literature are approximately 6 to $8 \mathrm{~K}$ for chronic irreversible tissue damage and 1.5 to $3 \mathrm{~K}$ for acute temperature-related neural activity and reversible inflammation. ${ }^{1,2}$ Assuming the validity of our predictions as a safety limit, we found many studies in the literature that stay below the thermal limit and do not overheat the tissue. Exceeding these limits may be biologically acceptable if tissue damage close to the fiber tip is acceptable or if the optical or heat dissipation characteristics of the structure of interest are significantly greater than the cortical surface (e.g., the structure is close to a ventricle). Caveats aside, a significant number of studies did exceed our predicted limits, ${ }^{18,19}$ further demonstrating the need to take heating into account in optogenetic experiments.

Apart from experimental measurements, most studies addressing the thermal effects of optogenetic stimulation have used finite-element Monte Carlo modeling of light spread in tissue to provide a bottom-up prediction. Our method takes the opposite approach, by modeling empirical data in a top-down manner. This approach critically requires accurate measurements of heating for accurate predictions. While finite-element modeling is the only approach that is able to predict the qualitative trends and three-dimensional shape of the heated area, the predicted results can show more than an order of magnitude difference from our empirical measurements (e.g., compare Fig. 2(a) with Fig. 5(c) from Ozden et al. $^{3}$ or the calculations from Stujenske et al. ${ }^{4}$ shown above). Furthermore, even qualitative predictions from finite-element models are not in agreement. Some Monte Carlo simulations show logarithmic-type temperature rises that are self-scaling in time ${ }^{3}$ and agree with our empirical results and model; in contrast, other Monte Carlo simulations predict saturating exponential-type temperature rises. ${ }^{4}$ Additionally, the simulations assume that biophysical input constants are inviolable physical values and therefore cannot take into account the significant animal-to-animal variability documented by our measurements. We believe that our empirical model permits much simpler calculation and empirical biological validation of predictions, with a minimal set of assumptions and limitations.

By fitting a model to experimentally measured heating in multiple animals (instead of using a bottom-up approach with universal biophysical parameters), we empirically circumvent the problem of local inhomogeneity in brain vascularization. For simulations, blood is typically factored in as a fixed absorption factor in each spatial element (however, see report by Azimipour et al. ${ }^{14}$ regarding early attempts to model this influence in a Monte Carlo model as well). As our data from individually illuminated vessels suggest, this homogeneity assumption is only valid for relatively large volumes that do not match the typically illuminated volumes. Experimental validation of worst-case scenarios (e.g., Fig. 4, directly illuminating over a blood vessel) is therefore a necessity. While our approach cannot fully describe the dynamics of laser brain heating as can 
(a)

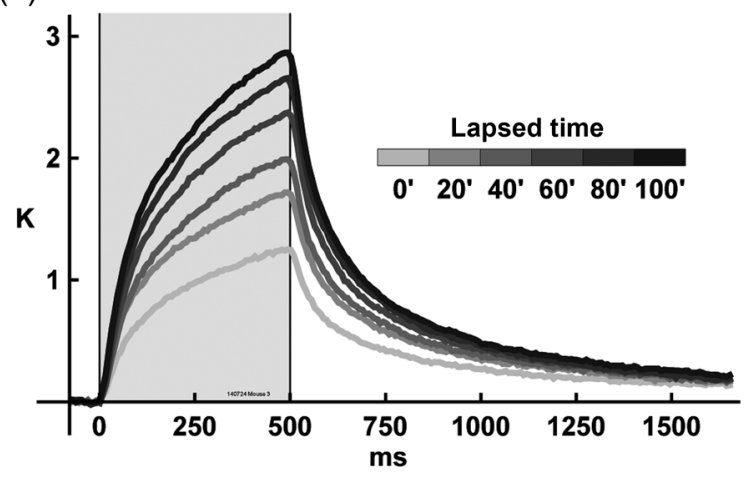

(b)

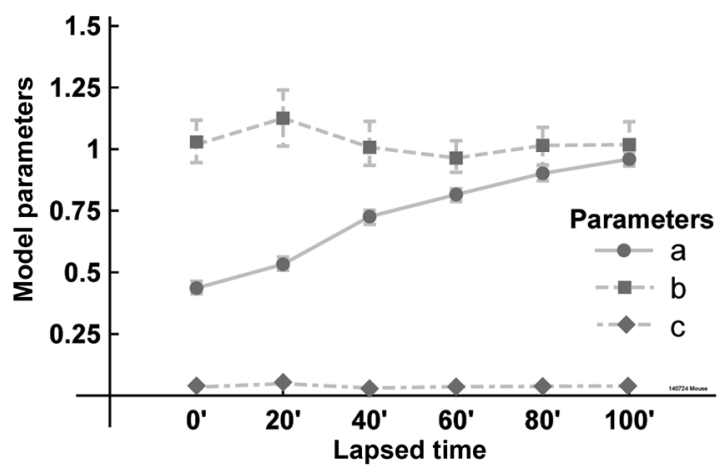

(c)

\begin{tabular}{|c|c|c|c|c|c|c|}
\hline Lapsed time $(\mathbf{m i n})$ & $\mathbf{0}$ & $\mathbf{2 0}$ & $\mathbf{4 0}$ & $\mathbf{6 0}$ & $\mathbf{8 0}$ & 100 \\
\hline $\mathbf{a}(1 \% \mathrm{Cl}-\mathbf{9 9} \% \mathrm{Cl})$ & $0.44(0.41-0.46)$ & $0.53(0.51-0.56)$ & $0.73(0.69-0.75)$ & $0.82(0.79-0.84)$ & $0.90(0.87-0.94)$ & $0.96(0.93-0.99)$ \\
\hline $\mathbf{b}(1 \% \mathrm{Cl}-99 \% \mathrm{Cl})$ & $1.02(0.94-1.12)$ & $1.13(1.01-1.24)$ & $1.01(0.93-1.11)$ & $0.96(0.91-1.03)$ & $1.01(0.93-1.09)$ & $1.02(0.94-1.11)$ \\
\hline $\mathbf{c}(1 \% \mathrm{Cl}-99 \% \mathrm{Cl})$ & $0.03(0.03-0.04)$ & $0.05(0.04-0.06)$ & $0.03(0.03-0.03)$ & $0.04(0.03-0.04)$ & $0.04(0.03-0.04)$ & $0.04(0.04-0.04)$ \\
\hline
\end{tabular}

(d)

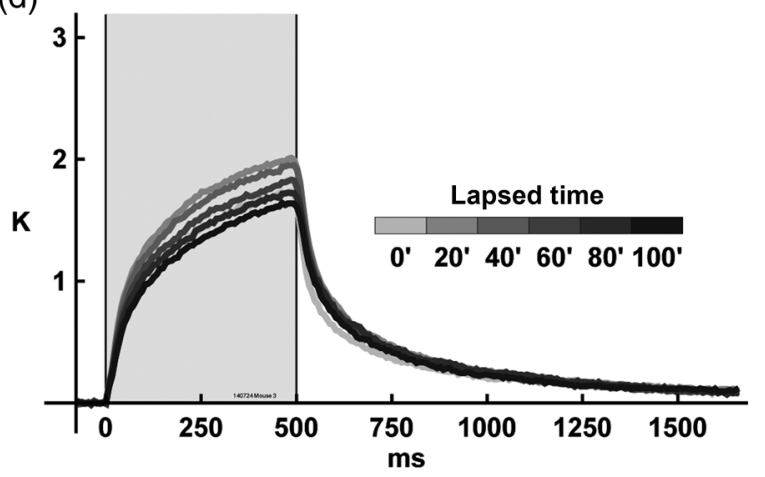

(e)

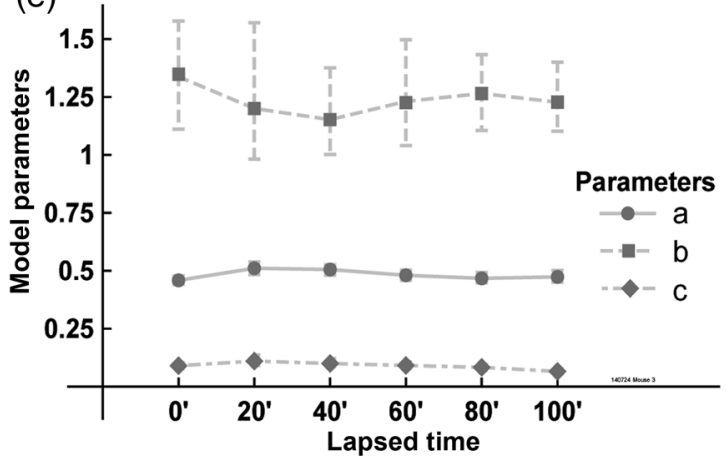

(f)

\begin{tabular}{|c|c|c|c|c|c|c|}
\hline Lapsed time (min) & 0 & 20 & 40 & 60 & 80 & 100 \\
\hline a $(1 \% \mathrm{Cl}-99 \% \mathrm{Cl})$ & $0.46(0.44-0.48)$ & $0.51(0.49-0.54)$ & $0.51(0.49-0.53)$ & $0.48(0.46-0.50)$ & $0.47(0.45-0.49)$ & $0.47(0.45-0.49)$ \\
\hline b (1\%Cl- 99\%Cl) & $1.34(1.11-1.59)$ & $1.20(0.98-1.50)$ & $1.17(1.02-1.37)$ & $1.23(1.05-1.48)$ & $1.25(1.10-1.43)$ & $1.22(1.09-1.37)$ \\
\hline c $(1 \% \mathrm{Cl}-99 \% \mathrm{Cl})$ & $0.09(0.08-0.10)$ & $0.11(0.09-0.13)$ & $0.10(0.09-0.12)$ & $0.09(0.08-0.11)$ & $0.08(0.07-0.10)$ & $0.07(0.06-0.08)$ \\
\hline
\end{tabular}

Fig. 6 Effect of time elapsed since craniotomy on tissue heating, for both $(a-c)$ dead and (d-f) alive mice. (a) The temperature increase in brain tissue from a dead animal due to optogenetic illumination grows higher as time passes after death. (b, c) This increase is reflected in the value of model parameter $\mathbf{a}$, whereas parameters a and $\mathbf{c}$ remain constant throughout time. (d) The temperature increase after illumination in brain tissue from a living animal remains constant in time. (e, f) Accordingly, none of the parameters for our model show trends in their variability.

the finite-element approach, the three parameters of our heating model do suggest biophysical correlates. Mathematically speaking, the two main parameters a and $\mathbf{c}$ describe the magnitude and kinetics of the heating process, respectively. Our model, therefore, constitutes a simple equation that can be interpreted in light of biophysical processes.

The strong light absorption of blood is likely also responsible for the equal heating for blue and green light that we found. For blue light, scattering is higher, but since hemoglobin has a local absorption minimum around $480 \mathrm{~nm}$, the increased absorption of green $532 \mathrm{~nm}$ light compensates for its lesser scattering. Only red light caused virtually negligible heating for common optogenetic illumination intensities. The development of red sensitive opsins ${ }^{20-22}$ will therefore lessen the risk of thermal damage in addition to provide better depth penetration.

In summary, our method offers a simple way to estimate actual temperature increases due to optogenetic stimulation with a variety of parameters. It can be applied easily to further anatomical brain structures and biological species, as well as the human brain. We hope that this will help to guide the design of complex and translational optogenetic stimuli as the method advances.

\section{Disclosures}

None of the authors have any conflicts of interest that might be construed to influence the results or interpretations within our manuscript. 


\section{Acknowledgments}

This work was funded by the Deutsche Forschungsgemeinschaft DFG (Priority Program 1665) and by fixed funds from the Leibniz Association. We would like to thank the two anonymous reviewers for their concise critiques and help in improving the manuscript.

\section{References}

1. W. Deng et al., "Optogenetics, the intersection between physics and neuroscience: light stimulation of neurons in physiological conditions," Am. J. Physiol. Regul. Integr. Comp. Physiol. 307, R1292-R1302 (2014).

2. S. Thomsen, "Pathologic analysis of photothermal and photomechanical effects of laser-tissue interactions," Photochem. Photobiol. 53, 825-835 (1991).

3. I. Ozden et al., "A coaxial optrode as multifunction write-read probe for optogenetic studies in non-human primates," J. Neurosci. Methods 219 , 142-154 (2013).

4. J. M. Stujenske, T. Spellman, and J. A. Gordon, "Modeling the spatiotemporal dynamics of light and heat propagation for in vivo optogenetics," Cell Rep. 12, 525-534 (2015).

5. K. Kuroda, "Non-invasive MR thermography using the water proton chemical shift," Int. J. Hyperthermia 21, 547-560 (2005).

6. M. T. Lippert et al., "Methods for voltage-sensitive dye imaging of rat cortical activity with high signal-to-noise ratio," J. Neurophysiol. 98 , 502-512 (2007).

7. A. S. Kalmbach and J. Waters, "Brain surface temperature under a craniotomy," J. Neurophysiol. 108, 3138-3146 (2012).

8. N. McAlinden et al., "Thermal and optical characterization of microLED probes for in vivo optogenetic neural stimulation," Opt. Lett. 38, 992-994 (2013).

9. G. Arias-Gil and K. Takagaki, GitHub Code Repository, 2015, http:// github.com/zalo23/doc-arias-gil-et-al-2015.

10. J. D. Johansson, "Spectroscopic method for determination of the absorption coefficient in brain tissue," J. Biomed. Opt. 15, 057005 (2010).

11. A. N. Yaroslavsky et al., "Optical properties of selected native and coagulated human brain tissues in vitro in the visible and near infrared spectral range," Phys. Med. Biol. 47, 2059-2073 (2002).

12. K. Takagaki, M. T. Lippert, and G. Arias-Gil, "Laser Heating App," 2015, http://www.lin-magdeburg.de/en/departments/systemphysiology/ neuro_optics/apps/laserheating/index.jsp.

13. W. F. Cheong, S. A. Prahl, and A. J. Welch, "A review of the optical properties of biological tissues," IEEE J. Quantum Electron. 26, 21662185 (1990).

14. M. Azimipour, F. Atry, and R. Pashaie, "Effect of blood vessels on light distribution in optogenetic stimulation of cortex," Opt. Lett. 40, 21732176 (2015).
15. J. A. Pearce, J. W. Valvano, and S. Emelianov, Optical-Thermal Response of Laser-Irradiated Tissue, pp. 399-453, Springer, Netherlands (2011).

16. I. N. Christie et al., "fMRI response to blue light delivery in the naive brain: implications for combined optogenetic fMRI studies," Neuroimage 66, 634-641 (2013).

17. J. Wells et al., "Biophysical mechanisms of transient optical stimulation of peripheral nerve," Biophys. J. 93, 2567-2580 (2007).

18. D. J. Calu et al., "Optogenetic inhibition of dorsal medial prefrontal cortex attenuates stress-induced reinstatement of palatable food seeking in female rats," J. Neurosci. 33, 214-226 (2013).

19. J. J. Nassi et al., "Optogenetic activation of normalization in alert macaque visual cortex," Neuron 86, 1504-1517 (2015).

20. J. Mattis et al., "Principles for applying optogenetic tools derived from direct comparative analysis of microbial opsins," Nat. Methods 9, 159-172 (2012).

21. B. D. Allen, A. C. Singer, and E. S. Boyden, "Principles of designing interpretable optogenetic behavior experiments," Learn Mem. 22, 232238 (2015).

22. F. Zhang et al., "Red-shifted optogenetic excitation: a tool for fast neural control derived from Volvox carteri," Nat. Neurosci. 11, 631-633 (2008).

Gonzalo Arias-Gil is a graduate student at the Leibniz Institute for Neurobiology (LIN) and the Otto-von-Guericke University in Magdeburg, Germany, where he trains under Dr. Kentaroh Takagaki and Frank W. Ohl. He received his master's degree in integrative neuroscience in 2015 from the Otto-von-Guericke University. He is studying the effects of transcranial direct-current stimulation in auditory learning using behavioral tasks and electrophysiology.

Frank Walter Ohl is a professor at the Otto-von-Guericke University in Magdeburg and a director at the LIN in Magdeburg. He received his $\mathrm{PhD}$ from the University of Darmstadt and continued to work at UC Berkeley and later at the LIN. His research interests are focused on the neuronal dynamics underlying learning.

Kentaroh Takagaki is a group leader at the LIN in Magdeburg, Germany. He received his PhD in 2007 and MD in 2010 from Georgetown University, training with Dr. Jian-Young Wu. He completed a Humboldt postdoctoral fellowship at the LIN with Dr. Frank W. Ohl and Dr. Henning Scheich. He uses high-sensitivity multichannel optical imaging and multichannel long-term electrophysiology to study emergent patterns in neural population activity associated with learning.

Michael Thomas Lippert is a group leader at the LIN in Magdeburg, Germany. He has previously worked at the Max-Planck-Institute for Biological Cybernectics in Tübingen and received his PhD in 2010 from the Otto-von-Guericke University in Magdeburg. He specializes in optical techniques to measure and influence neuronal activity. 